OPEN ACCESS

Edited by:

Hansen Wang,

University of Toronto, Canada

Reviewed by:

Laurie Doering,

McMaster University, Canada Lucia Ciranna,

Università di Catania, Italy

*Correspondence:

Dalit Ben-Yosef

dalitb@ttvmc.gov.il

${ }^{\dagger}$ Present Address:

Michael Telias,

Department of Molecular and Cell

Biology, University of California at

Berkeley, USA

Received: 04 February 2016 Accepted: 26 April 2016 Published: 12 May 2016

Citation:

Telias M, Segal M and Ben-Yosef D (2016) Immature Responses to GABA

in Fragile $X$ Neurons Derived from Human Embryonic Stem Cells. Front. Cell. Neurosci. 10:121. doi: 10.3389/fncel.2016.00121

\section{Immature Responses to GABA in Fragile $X$ Neurons Derived from Human Embryonic Stem Cells}

\author{
Michael Telias ${ }^{1,2 \dagger}$, Menahem Segal ${ }^{3}$ and Dalit Ben-Yosef ${ }^{1,2 *}$ \\ 1 Wolfe PGD-Stem Cell Lab, Racine IVF Unit, Tel-Aviv Sourasky Medical Center, Lis Maternity Hospital, Tel-Aviv, Israel, \\ ${ }^{2}$ Department of Cell and Developmental Biology, Sackler Medical School, Tel-Aviv University, Tel-Aviv, Israel, ${ }^{3}$ Department of \\ Neurobiology, The Weizmann Institute of Science, Rehovot, Israel
}

Fragile $X$ Syndrome $(F X S)$ is the most common form of inherited cognitive disability. However, functional deficiencies in FX neurons have been described so far almost exclusively in animal models. In a recent study we found several functional deficits in FX neurons differentiated in-vitro from human embryonic stem cells (hESCs), including their inability to fire repetitive action potentials, and their lack of synaptic activity. Here, we investigated the responses of such neurons to pulse application of the neurotransmitter GABA. We found two distinct types of responses to GABA and sensitivity to the GABA-A receptor antagonist bicuculline; type 1 (mature) characterized by non-desensitized responses to GABA as well as a high sensitivity to bicuculline, and type 2 (immature) which are desensitized to GABA and insensitive to bicuculline. Type 1 responses were age-dependent and dominant in mature WT neurons. In contrast, FX neurons expressed primarily type 2 phenotype. Expression analysis of GABA-A receptor subunits demonstrated that this bias in human FX neurons was associated with a significant alteration in the expression pattern of the GABA-A receptor subunits $\alpha 2$ and $\beta 2$. Our results indicate that FMRP may play a role in the development of the GABAergic synapse during neurogenesis. This is the first demonstration of the lack of a mature response to GABA in human FX neurons and may explain the inappropriate synaptic functions in FXS.

Keywords: Fragile $\mathrm{X}$ syndrome, GABA, neural development, human embryonic stem cells, in-vitro neural differentiation

\section{INTRODUCTION}

Fragile X syndrome (FXS) is the most common form of inherited intellectual disability (Penagarikano et al., 2007; Bagni and Oostra, 2013). Individuals with FXS show cognitive disabilities, as well as high-comorbidity with autism and epilepsy. It is caused by developmentally regulated silencing of the FMR1 gene, and absence of its encoded protein, FMRP (Willemsen et al., 2002). Loss of FMRP impairs neurogenesis of adult neural stem cells in mice (Luo et al., 2010; Guo et al., 2011) as well as embryonic neurogenesis of human pluripotent stem cells and neural precursor cells (Eiges et al., 2007; Adusei et al., 2010; Sheridan et al., 2011; Telias et al., 2013, 2015b).

In FX neurons obtained from $f m r 1^{-1-}$ mice, the absence of the FMRP protein has been linked to an aberrant response to either the glutamate or the gamma-aminobutyric acid (GABA) neurotransmitter (Bear et al., 2004; Braat and Kooy, 2015). Several studies in $f m r 1^{-/-}$mice have demonstrated a link between the GABAergic inhibitory system and the impaired neuronal activity 
observed in FXS (Braat and Kooy, 2015), in which abnormal expression of GABAergic proteins in different brain areas and during different developmental stages has been shown (Adusei et al., 2010; Kratovac and Corbin, 2013). During development, neurons first respond to GABA with excitation and later switch to inhibition (Ben-Ari, 2015). It was later reported that such developmental switch is delayed in $f m r 1^{-/-}$mice (He et al., 2014). However, this hypothesis has not been tested in human FX neurons, mainly due to the lack of suitable research models. Therefore, analysis of the functional and molecular expression of the GABArgic synapse in-vitro in FX human neurons can shed new light on the involvement of inhibitory transmission in FXS.

We were able to derive FX human embryonic stem cells (hESCs) carrying the naturally occurring full mutation $(>200$ CGG repeats) at the FMR1 locus, and demonstrated that in-vitro neural differentiation effectively produces neurons that gradually lose FMRP, and consequently demonstrate impaired neurogenesis (Telias et al., 2013). Recently, we have shown that this gradual downregulation of FMRP during neurogenesis until its complete absence in mature neurons leads to impaired electrophysiological properties, including lack of repetitive action potential firing, reduced neurotransmitter release and poor synaptogenesis (Telias et al., 2015a). In the present study we analyzed the functional response of these FX human neurons to GABA. Our results show that hESC neurons produce two types of responses to GABA, a mature (type 1) and an immature (type 2) response: in wild-type (WT) neurons, the immature responses are gradually replaced by the mature form, but in FX cells, type 2 responses are persistent. In addition, human FX neurons have increased RNA levels of $G A B A-A \alpha 2$ and reduced RNA levels of $G A B A-A \beta 2$. Taken together our results indicate that lack of FMRP may affect the composition of the GABA-A receptors and prevents their maturation.

\section{MATERIALS AND METHODS}

\section{Human Embryonic Stem Cells and In-vitro Neural Differentiation}

The use of spare IVF-derived embryos diagnosed by Preimplantation Genetic Diagnosis (PGD) for the derivation of hESCs was approved by the Israeli National Ethics Committee (7/04-043). Three Fragile X human embryonic stem cell (hESC) lines with $>200$ CGG repeats in the FMR1 gene were studied: HEFX1 (male) and Lis_FX6 (male), derived at TelAviv Sourasky Medical Center and SZFX6 (male), derived in Shaare Tzedek Medical Center. These three lines were entitled here systematically: FX1, FX2, and FX3, and were all fully characterized previously (Eiges et al., 2007; Telias et al., 2013). The hESC line HUES-13 (male; kindly provided by the Melton Lab, Harvard University), was previously shown by us to include $<30$ CGG repeats in the FMR1 gene (Telias et al., 2013) and was used here as WT. hESCs were cultured on feeder layers of mitomycin C (Sigma)-inactivated mouse embryonic fibroblasts (MEF) in hES-medium supplemented with bFGF (R\&D), as we previously described (Telias et al., 2013, 2015a). Before induction of in-vitro neural differentiation, hESCs were cultured on Matrigel (BD)-coated wells for two passages. The dual SMAD inhibition protocol was applied as we previously described (Telias et al., 2014, 2015a). Briefly, neural induction was achieved by gradually changing the medium from hES to N2 while adding dorsomorphin and SB431542 for 10 days; for neuronal induction the medium was changed to N2/B27 supplemented with BDNF, GDNF, ascorbic acid, dbcAMP and DAPT for 10 additional days. At day 20 cells were dissociated using Accutase (Life Tech.) and seeded on $13 \mathrm{~mm}$ glass coverslips pre-coated with $50 \mu \mathrm{g} / \mathrm{ml}$ Poly-D-Lysine and $20 \mu \mathrm{g} / \mathrm{ml}$ Laminin (Sigma) at a seeding density of $\sim 1.0 \times 10^{5} \mathrm{cells} / \mathrm{cm}^{2}$. From day 20 , neurons were continuously grown in N2/B27 medium supplemented with $20 \mathrm{ng} / \mathrm{ml}$ BDNF, GDNF, and NT3, unless otherwise specified. Concentrations of reagents and growth factors used were as follows: $5 \mu \mathrm{M}$ dorsomorphin (Stemgent), $10 \mu \mathrm{M}$ SB431542 (Stemgent), $20 \mathrm{ng} / \mathrm{ml}$ BDNF (PeproTech), $20 \mathrm{ng} / \mathrm{ml}$ GDNF (PeproTech), $0.2 \mathrm{mM}$ ascorbic acid (Sigma), $0.5 \mathrm{mM}$ dbcAMP (Sigma), $10 \mu \mathrm{M}$ DAPT (Tocris), $20 \mathrm{ng} / \mathrm{ml}$ NT3 (PeproTech). N2 medium was composed of DMEM:F12 (Life Tech.), supplemented with 1\% N2 (Life Tech.), 1\% non-essential amino acids (BioInd.), 1\% Glutamax (Life Tech.), and $100 \mu \mathrm{g} / \mathrm{ml}$ Primocin (InvivoGen). N2/B27 Medium was a 1:1 mixture of $\mathrm{N} 2$ and B27 media. B27 medium was composed of Neurobasal (Life Tech.), supplemented with 1\% B27 (Life Tech.), 1\% nonessential amino acids (BioInd.), 1\% Glutamax (Life Tech.), and $100 \mu \mathrm{g} / \mathrm{ml}$ Primocin (InvivoGen). Differentiation was carried out three times for each hESC line, cells were analyzed between days 40 and 160 .

\section{Electrophysiology and Pharmacology}

Recordings were conducted as previously described (Telias et al., 2013, 2014, 2015a). In brief, neurons differentiated on glass coverslips were transferred to a recording chamber in standard recording medium, containing (in $\mathrm{mM}$ ):10 $\mathrm{HEPES}, 4 \mathrm{KCl}, 2$ $\mathrm{CaCl}_{2}, 1 \mathrm{MgCl}_{2}, 139 \mathrm{NaCl}, 10 \mathrm{D}$-glucose (340 mOsm, $\mathrm{pH}$ 7.4). Cells were patch-clamped with pipettes containing (in $\mathrm{mM}$ ) 136 K-gluconate, $10 \mathrm{KCl}, 5 \mathrm{NaCl}, 10 \mathrm{HEPES}, 0.1$ EGTA, $0.3 \mathrm{Na}-\mathrm{GTP}$, $1 \mathrm{Mg}$-ATP, and 5 phosphocreatine, $\mathrm{pH} 7.2$ (pipette tip resistance was 5-8 M $\Omega$ ). Membrane potential was held at $-60 \mathrm{mV}$. GABA ( $2 \mathrm{mM}$ in the pressure pipette, tip diameter $2-4 \mu \mathrm{m}$ ) was applied within $10 \mu \mathrm{m}$ from the recorded neuron for a duration of $50 \mathrm{~ms}$. Signals were amplified with a Multiclamp700B amplifier and recorded with Clampex 9.2 software (Axon Instruments). Bicuculline (Sigma) was applied at a concentration of $20 \mu \mathrm{M}$ into the recording bath.

\section{Quantitative Real Time PCR}

Relative transcription levels were analyzed using quantitative Real Time PCR (qRT-PCR) on cDNA reversed transcribed from RNA samples. RNA extracted from cells (RNAeasy Mini Kit-Qiagen), was stored at $-80^{\circ} \mathrm{C}$ with RNAse Inhibitor (Roche) and DNAseI (Roche). RNA (100 ng) was reversed transcribed with Super Script III RT-PCR kit (Invitrogen). Quantitative Real Time PCR (qRT-PCR) was performed using SYBR Green (ABgene). Cycling and analysis were performed using Rotor Gene 6000 Series (Corbett) and its complementary analysis software. The house keeping gene GAPDH was used 
as a control for $\Delta \Delta \mathrm{Ct}$ analysis of results. All qRT-PCR assays included Non-Template Control (NTC), and adult human-FXS white blood cells. Oligo DNAs (custom made primers) were purchased from Sigma, and their sequences are as follows: GAPDH, Fwd: AGCCACATCGCTCAGACA CC, Rev: ATACGACCAAATCCGTTGACTC; Gephyrin, Fwd: ATGATCCTTACTAACCACGACCA, Rev: AGATTTATCCCA CTGCGGTCTT; GABA-A $\alpha$, Fwd: GGAAGAAGCTATGGA

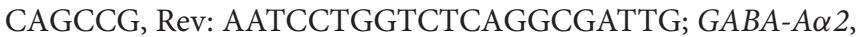
Fwd: GCCAATCAATCGGAAAGGAGAC, Rev: TTCCCATCC CAAGTCCATCCTC; GABA-A $\beta 2$, Fwd: TGCCTGATACCTATT TCCTGAACG, Rev: GATTCCTAATGCCACCCTTGC; GABA$A \gamma 2 S$, Fwd: TGCACACTCATTGTCGTCCTATCCTGG, Rev: TTAAACAGGCAGAAGGCAGTGGGG; GABA-A $\delta$, Fwd: AGG ACATCGTCTACTACTGGTCGGAGAG, Rev: TCGGCGTTG AAATGAGCAAAGG.

\section{Immunofluorescence}

Immunostaining was performed as described previously (Telias et al., 2013, 2015b). Briefly, cells were fixed for $15 \mathrm{~min}$ at room temperature (RT) using Cytofix (BD Biosciences) and washed with PBS. Primary antibodies were incubated overnight at $4^{\circ} \mathrm{C}$, in a PBS solution containing $2.5 \%$ BSA and $0.1 \%$ Triton. Staining with secondary antibody was performed for $1 \mathrm{~h}$ at RT in the dark. Primary antibodies, mouse anti-human TUJ1 (Millipore), and rabbit anti-human GAD 65 (Santa Cruz), were detected using sheep anti-mouse Cy2-conjugated or goat anti-rabbit Cy3conjugated secondary antibodies (Jackson Laboratories). Nuclei were stained with DAPI (Sigma-Aldrich). Images were taken using a Zeiss LSM 700 confocal microscope.

\section{Data Analysis}

Data from patch clamp recordings were subjected to a $500-\mathrm{Hz}$ low-pass filter and analyzed using Clampfit-9 and SigmaPlot. Statistical analysis was performed using SigmaPlot and online GraphPad QuickCalcs (http://www.graphpad.com/quickcalcs). Difference level of $<0.05$ was considered significant.

\section{RESULTS}

\section{Responses to GABA in Neurons Differentiated In-vitro from Human Embryonic Stem Cells}

The responses of FX and WT neurons to GABA were examined between days 40 and 160 of in-vitro neural differentiation, carried out three times in three FX-hESC lines (designated FX1, FX2, and FX3) and one WT-hESC line (see Section Materials and Methods and Figure 1A). As we previously reported, both WTand FX-derived neurons express the neuronal proteins NeuN, CaMK-II, TUJ1, and MAP2, but FX neurons do not express FMR1 or FMRP (Telias et al., 2015a). Here we show that already at day 40 of differentiation, both WT and FX neurons, express
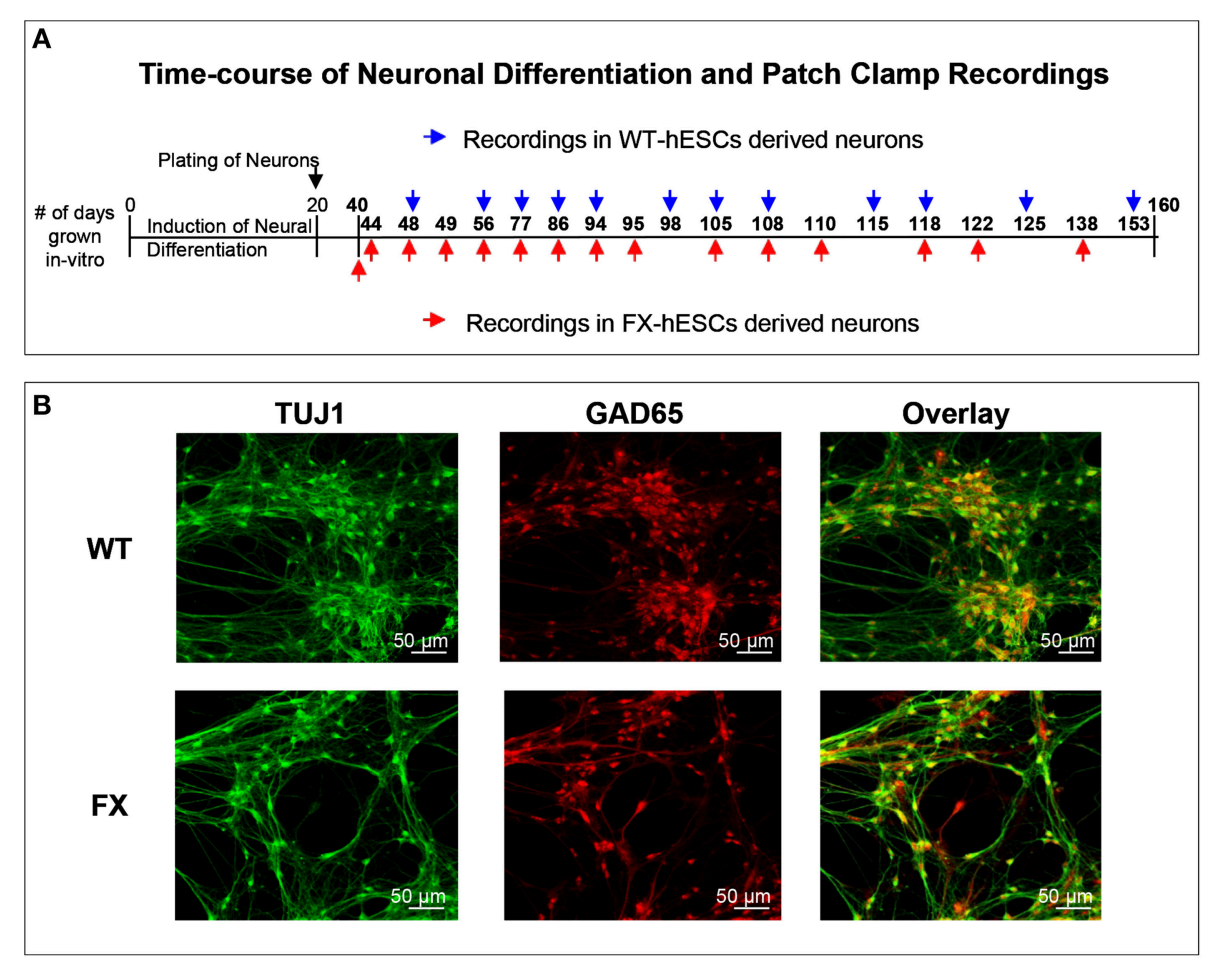

FIGURE 1 | (A) Outline of the time-course of neuronal differentiation and the time-points at which electrophysiological recordings were carried out in WT (blue) and FX (red) human embryonic stem cells-derived neurons. (B) Immunofluorescence images for cellular localization of TUJ1 (green, left), GAD65 (red, middle) and their overlay (yellow, right); in WT (top row), and FX (bottom row) 40-days old neurons. Scale is $50 \mu \mathrm{m}$. 
co-localized GAD65 (glutamic acid decarboxylase 65) and TUJ1, indicating that these neurons have the enzymatic machinery necessary for producing and secreting GABA (Figure 1B). From these immunostaining assays we could estimate that $>\sim 90 \%$ of TUJ1-positive cells were also positively stained for GAD65. We have already shown that critical differences in firing patterns and synaptic activity exist between FX and WT neurons (including impaired action potential firing and reduced spontaneous synaptic activity) during the same time frame of differentiation considered in the present study (Telias et al., 2015a). In the present study we analyzed the electrophysiological responses of WT and FX neurons to topical application of GABA. At several time-points during differentiation (Figure 1A-blue and red arrows), we carried-out patch clamp recordings on WT and FX-hESC derived neurons. Voltage-clamped cells, held at $-60 \mathrm{mV}$ were exposed to a 50 -ms, $2 \mathrm{mM}$ GABA applied through a pressure pipette. Two distinct responses to GABA were found in both WT and FX neurons, tentatively designated herein as "type 1" and "type 2." A total of 30 WT neurons were recorded as compared to 35 FX neurons (FX1 $n=11$, FX2 $n=9$, FX3 $n=15$ ), in three differentiation experiments (Figures $\mathbf{2 A}, \mathbf{B}$ ). Type 1 responses were characterized as persistent and nondesensitized inward currents to successive pulse application of GABA. In contrast, type 2 responses showed time-dependent desensitization of reactivity to GABA, such that a consecutive GABA pulse applied $\sim 1 \mathrm{~s}$ later resulted in no response, whereas 30 s later a $\sim 80 \%$ reduction in the peak amplitude of the recorded current was still evident, and a full recovery was found more than $120 \mathrm{~s}$ after the first response (Figure 2A). However, both type 1 and 2 responses showed similar reversal potentials (Er), between -30 and $-20 \mathrm{mV}$ at an equivalent age (Figure 2B). In addition, the two types differed in their sensitivity to the GABAA receptor antagonist bicuculline (BIC, at $20 \mu \mathrm{M}$; Figure 2C). In type 1 responses, BIC abolished GABA induced currents in all cells tested (WT $n=6, \mathrm{FX} n=1$ ), while type 2 responses were insensitive to BIC (WT $n=5, \mathrm{FX} n=8$ ). It is important to note that hyperpolarization of cells to $-100 \mathrm{mV}$ speeded up recovery from desensitization by $\sim 50 \%$ in both WT and FX neurons (WT $n=3$, FX $n=4$ ), and that none of the cells analyzed, WT or FX, responded to pressure application of the GABA-B agonist baclofen (10-20 $\mu \mathrm{M}$; WT $n=7$, FX $n=6)$, indicating that the responses are of GABA-A type.

\section{Age Distribution of Type1 and Type2 in WT and FX}

Next, we analyzed the frequency distribution of type 1 vs. type 2 responses, in FX and WT neurons. Our results show that $37 \%$ of WT neurons ( $n=11$ of 30$)$ were classified as having type 1 responses, while $63 \%(n=19$ of 30$)$ were classified as type 2 (Figure 3A). In contrast, in FX neurons, only $9 \%(n=$ 3 of 35, all 3 from FX3 line only) were classified as type 1, while $91 \%(n=32$ of 35$)$ were of type 2 . This bias of FX neurons toward type 2 responses was statistically significant $(p<$ 0.01 ). Furthermore, within type 2 responses, time-dependent re-sensitization to GABA was significantly decreased in FX neurons as compared to WT (Figure 3B), suggesting that FX

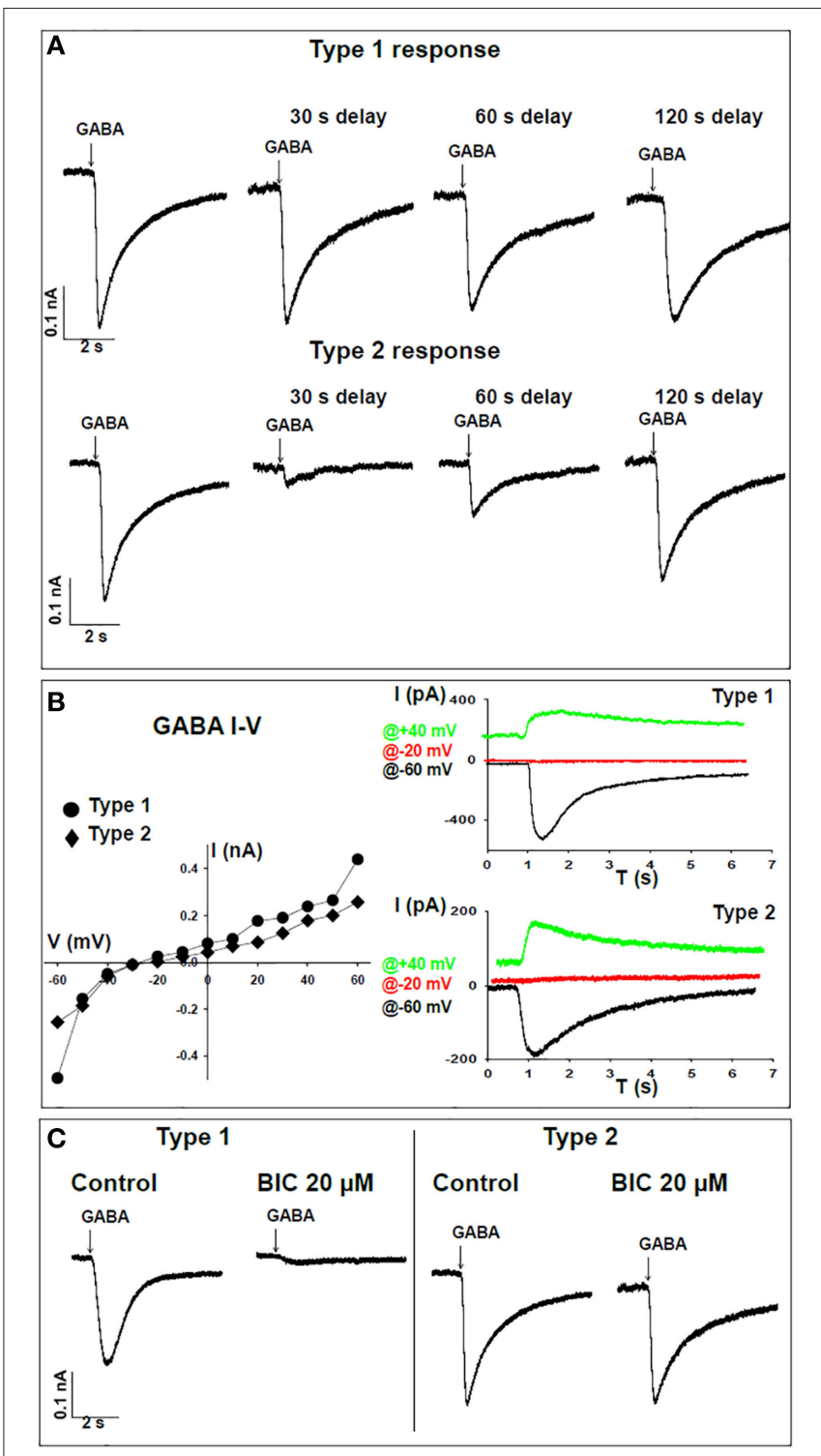

FIGURE 2 | Time-dependent responses of WT neurons to GABA.

(A) Representative traces of voltage-clamp recordings for time-dependent responses to GABA demonstrating "type 1" (top) and "type 2" (bottom) patterns. Cells were held at $-60 \mathrm{mV}$ and $2 \mathrm{mM}$ GABA was applied by pressure from an adjacent pipette. Recordings were taken for the first puff and following consecutive delays of 30,60, and $120 \mathrm{~s}$. (B) Left panel, I-V curve for type 1 (circles) and type 2 (diamonds) GABA responses in single neurons. Right panel, representative traces corresponding to the same neuron shown on the left. Traces are sown for recordings at $-60 \mathrm{mV}$ (black), $-20 \mathrm{mV}$ (red), and $+40 \mathrm{mV}$ (green). (C) Representative traces of voltage-clamp recordings showing sensitivity to $20 \mu \mathrm{M}$ bicuculline (BIC) in type $1(\mathrm{WT} n=6, \mathrm{FX} n=1)$ and lack of sensitivity to BIC in type $2(\mathrm{WT} n=5, \mathrm{FX} n=8)$ responses to GABA.

neurons have a higher tendency to a type 2-like response, with increased desensitization in reaction to GABA and reduced capability for re-sensitization. More importantly, the distribution of both types of response was age-dependent in WT but not in FX neurons (Figure 3C). Type 1-WT neurons were older, with an in-vitro age ranging from 86 to 153 days, while type 2-WT 

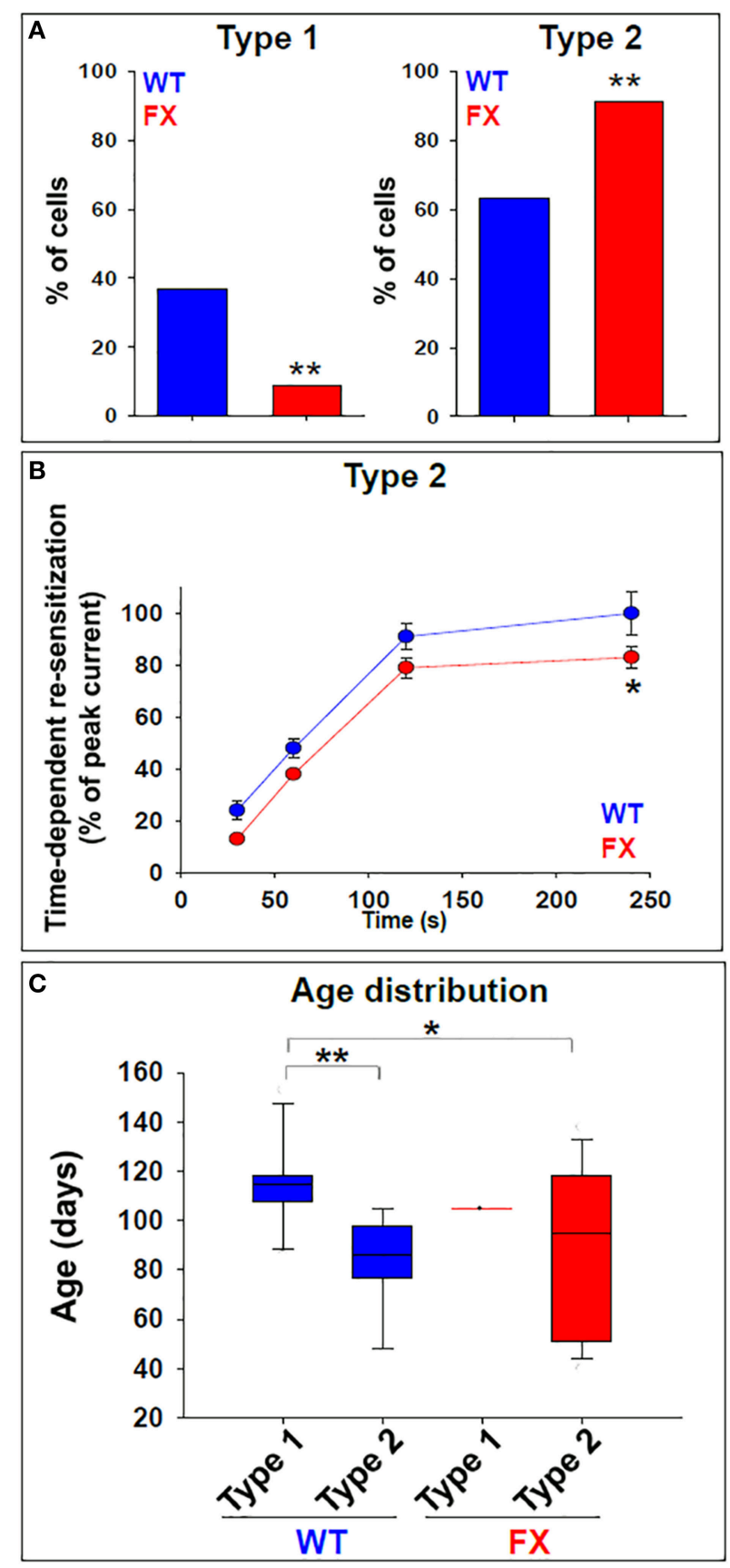

FIGURE 3 | GABA responses in WT and FX neurons. (A) Total incidence of type 1 and type 2 responses to GABA in WT (blue) and FX (red) neurons. Values are the percent of cells in each category out of the total number of cells examined. WT $n=30$, FX $n=35$ (FX1 $n=11$, FX2 $n=9$, FX3 $n=15) .{ }^{* *} p<0.01$, Chi-square test. (B) Quantification of time-dependent re-sensitization in type 2 responses, in WT (blue) and FX (red) neurons. Values are presented as percent of the peak current obtained with the first GABA pulse (before desensitization). WT $n=30$, FX $n=35$ (FX1 $n=11$, FX2 $n=9$, FX3 $n=15)$. Values are mean \pm SEM. ${ }^{*} p<0.05$, $t$-test. (C) Box plot showing distribution of type 1 and type 2 responses in relation to neuronal in-vitro age following differentiation induction, in WT (blue) and FX (red) neurons. WT $n=30$, FX $n=35$ (FX1 $n=11$, FX2 $n=9$, FX3 $n=15) .{ }^{*} p<0.05,{ }^{*} p<0.01$, Chi-square test. See also Table 1 for details. neurons were younger, with an in-vitro age ranging from 48 to 105 days (Table 1). This difference in age distribution between type 1 and type 2 response in WT was statistically significant $(p<0.05)$. In contrast, no age-dependency was found in FX neurons. Only three cells were classified as type 1-FX, which were all 105 days old and derived from line FX3 only. In contrast, type 2-FX neurons showed an age distribution ranging from day 40 to 138 , which was significantly different from type 1WT, but not from type 2-WT or type 1-FX (Table 1). Therefore, we conclude that type 2 response to GABA corresponds to an immature neuronal phenotype, which develops into the mature type 1 response. The strongly-biased distribution of FX neurons toward type 2 suggests that FMR1 downregulation induced a significant delay in the transition from an immature to a mature neuronal phenotype, highlighting the important role played by FMRP in human embryonic neuro-development in general, and in the process of "GABA maturation" in particular. In our recently published study on the electrophysiological deficiencies of human FX neurons derived from hESCs (Telias et al., 2015a), we showed that lack of spontaneous synaptic activity in FX cells was rescued by co-culture with rat hippocampal neurons (Figure S1A). However, co-culture did not reverse the type 2 response to GABA in FX neurons (Figure S1B), further emphasizing the idea that FMRP may exert a direct effect on GABA-A receptor composition.

\section{Expression of GABA-A Receptor Subunits in WT and FX Neurons}

In order to elucidate the molecular mechanism responsible for the biased tendency of FX neurons toward type 2 responses, we analyzed the expression of Gephyrin (which is specifically expressed in GABAergic neurons) and several subunits of the GABA-A receptor. RNA was extracted from WT and FX neurons at 120-130 days of differentiation, when most WT neurons respond to GABA as type 1, but FX neurons are characterized as type 2 (Figure 3C). We found that WT and FX neurons express

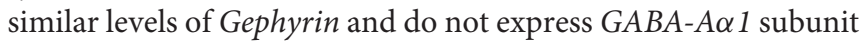
(data not shown). In contrast, FX neurons expressed significantly higher levels of $G A B A-A \alpha 2$ subunit (WT $=100 \pm 22.5 \%$; FX $=139.6 \pm 9.8 \% ; p<0.05)$, and significantly lower levels of GABA-A $\beta 2$ subunit $(\mathrm{WT}=100 \pm 10 \%$; FX $=17.7 \pm 1.8 \%$; $p<0.01$; Figure 4). Non-significant differences in expression were found in $G A B A-A \gamma 2 S$ (WT $=100 \pm 9.3 \%$; $\mathrm{FX}=75.3 \pm$ $15.8 \% ; p>0.05)$, and in $G A B A-A \delta$ subunit $(\mathrm{WT}=100 \pm 11.5 \%$; $\mathrm{FX}=107.1 \pm 10.8 \% ; p>0.05)$. These results suggest that the significant increase in the mRNA expression of $\alpha 2$ subunit and the robust reduction in the mRNA expression of $\beta 2$ subunit in FX neurons could be responsible for the persistence of the immature type- 2 response in FX lines, and hint to a possible developmentally regulatory role for FMRP in the expression of specific GABA-A subunits.

An increase in $\alpha 2$ expression has been linked to slower decay time in GABA-induced currents both in-vivo and invitro (Okada et al., 2000; Dixon et al., 2014). Therefore, we analyzed the value of tau in GABA responses in WT and FX neurons (Figure 5A). Our analysis shows a statistically significant 
TABLE 1 | Full data for age distribution.

\begin{tabular}{|c|c|c|c|c|c|c|c|}
\hline \multicolumn{4}{|c|}{ WT } & \multicolumn{4}{|c|}{ FX } \\
\hline No. of cells & Age (days) & No. of cells & Age (days) & No. of cells & Age (days) & No. of cells & Age (days) \\
\hline 1 & 86 & 1 & 48 & 1 & 105 & 1 & 40 \\
\hline 2 & 98 & 2 & 48 & 2 & 105 & 2 & 40 \\
\hline 5 & 115 & 5 & 77 & & & 5 & 44 \\
\hline 6 & 115 & 6 & 77 & & & 6 & 48 \\
\hline 7 & 118 & 7 & 86 & & & 7 & 49 \\
\hline 8 & 118 & 8 & 86 & & & 8 & 49 \\
\hline 9 & 118 & 9 & 86 & & & 9 & 56 \\
\hline & & 14 & 95 & & & 14 & 94 \\
\hline & & 15 & 98 & & & 15 & 94 \\
\hline & & 16 & 98 & & & 16 & 95 \\
\hline & & 17 & 105 & & & 17 & 95 \\
\hline & & 18 & 105 & & & 18 & 95 \\
\hline & & 19 & 108 & & & 19 & 108 \\
\hline & & & & & & 20 & 108 \\
\hline & & & & & & 21 & 108 \\
\hline & & & & & & 22 & 110 \\
\hline & & & & & & 23 & 110 \\
\hline & & & & & & 31 & 138 \\
\hline & & & & & & 32 & 138 \\
\hline Mean & 114.73 & Mean & 83.63 & Mean & 105 & Mean & 89.81 \\
\hline Sem & 5.04 & Sem & 4.54 & Sem & 0 & Sem & 5.76 \\
\hline$n$ & 11 & $n$ & 19 & $n$ & 3 & $n$ & 32 \\
\hline
\end{tabular}

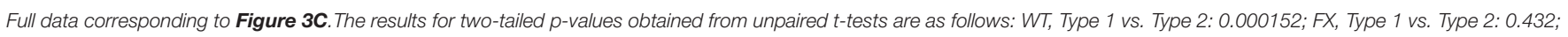

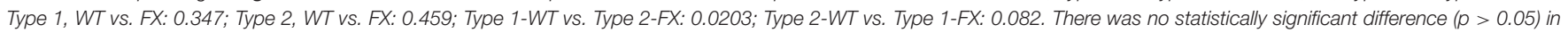
the age range of WT neurons ([48-153], $n=30$ ) as compared to the age range of FX neurons ([40-138], $n=35)$.

increase in tau (i.e., slower mono-exponential decay) in FX neurons displaying type 2 responses as compared to type 1 responses in WT and FX (both $p<0.01$; Figure 5B). Tau had a non-significant tendency to increase in type 2 responses in WT neurons as compared to type $1-\mathrm{WT}(p=0.12)$ or as compared to type 1 -FX $(p=0.06)$, and to decrease as compared to type 2-FX ( $p=0.07)$. These results suggest that an increase in $\alpha 2$ mRNA in FX cells could be responsible for the immature response to GABA observed in the vast majority of FX neurons.

\section{DISCUSSION}

In this study we examined the effect of the FMR1 mutation on the functional and molecular aspects of the GABAergic system in human neurons. We have shown that WT neurons obtained by in-vitro neural differentiation of hESCs undergo an agedependent phase in which they exhibit a non-classical response to GABA, characterized by time-dependent desensitization and lack of sensitivity to GABA-A antagonist, bicuculline. We show that, while this immature response is transient in WT neurons, it is 


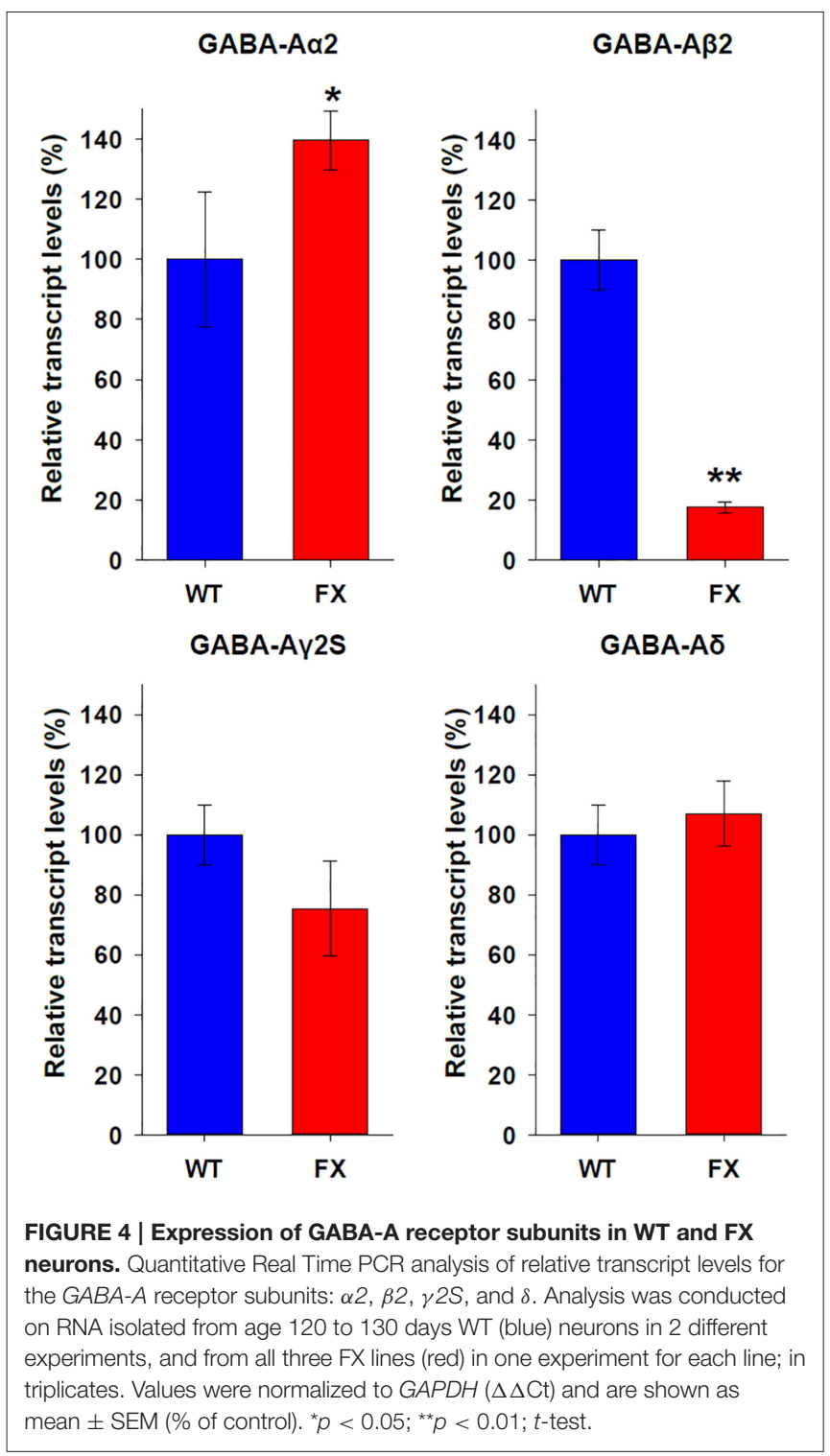

persistent across FX lines and throughout development. In order to unveil the molecular mechanism behind this phenomenon, we analyzed the transcription of specific $G A B A-A$ receptor subunits and found an increase in the mRNA expression of the $\alpha 2$ subunit and a robust reduction in the mRNA expression of the $\beta 2$ subunit. These changes in the expression of $G A B A-A$ receptor subunits might be associated with an increase in GABA-response decay time. Our results suggest a role for FMRP in regulating the maturation of GABAergic neurotransmission, characterized here for the first time in human neurons.

\section{Abnormal GABAergic Transmission Associated with Fragile $X$ Neurodevelopment}

It has been previously established that GABA-A receptors are under-expressed in the hippocampus and cortex of $f \mathrm{mrl}^{-/-}$ mice (D’Hulst and Kooy, 2009; Braat and Kooy, 2015). Most

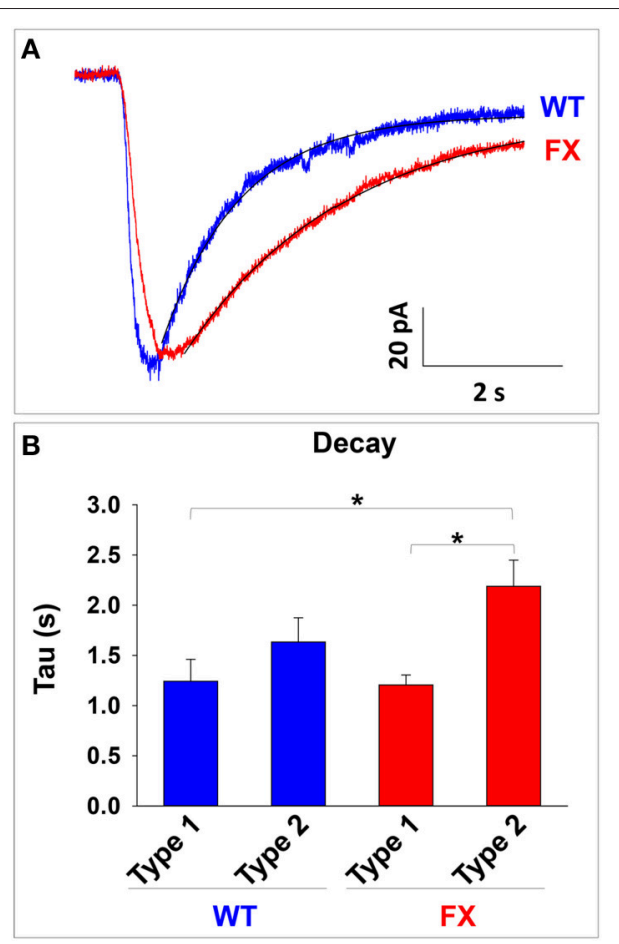

FIGURE 5 | Analysis of GABA response decay in WT and FX neurons. (A) Representative traces of GABA responses and the exponential curve fit used to calculate the value of the decay. A trace from a type 1 response in WT is shown in blue and a trace from a type 2 response in FX is shown in red. Fit curves are shown in black. (B) Measurement of the decay (Tau) in WT and FX neurons, for type 1 and type 2 responses. Values are shown as mean $\pm \mathrm{SEM}$. ${ }^{*} p<0.01$, ANOVA.

importantly, it has been demonstrated that FMRP directly binds mRNA of several GABA-A receptor subunits (Braat et al., 2015). GABAergic transmission plays a crucial role during neurodevelopment, in which it switches from excitatory to inhibitory action due to changes in the intracellular chloride concentration, affecting the development of neurons at various levels, including their proliferation, migration, and dendritic arborization (Ben-Ari et al., 2012). It has been recently reported that in $\mathrm{fmrl}^{-/-}$mice, this developmental switch from excitation to inhibition is delayed ( $\mathrm{He}$ et al., 2014). Indeed, lack of FMRP in developing cortical neurons increases the expression of the $\mathrm{Na}^{+}-\mathrm{K}^{+}-\mathrm{Cl}^{-}$co-transporter, delaying the hyperpolarization of $\mathrm{Cl}^{-}$reversal potential. In this study, we have uncovered two distinct types of responses to GABA in human neurons: while some of the neurons showed the expected response to GABA (including consecutive responses to repeated exposures, which were abolished by bicuculline), other neurons responded with an atypical time-dependent desensitization to consecutive GABA applications and complete insensitivity to bicuculline. More importantly, we have shown the relevance of this specific response in the context of human neurogenesis and FXS: while this atypical response eventually disappears with age in control neurons, it persists in FX neurons. These results suggest a new mechanism for the developmental delays and cellular 
abnormalities observed during neurodevelopment of FX-hESCS, concomitant with gradual downregulation of FMR1.

Desensitization of GABA-A receptors has already been described in cultured hippocampal rodent neurons (Segal, 1993; Jones and Westbrook, 1995). Most importantly, such desensitization was associated with younger age (1-7 daysold) and disappeared with more advanced age (21-28 daysold; Segal, 1993). Expression assays in oocytes and HEK cells have produced data indicating that subunit composition directly affects the desensitization properties of GABA-A receptors (Martinez-Torres et al., 2000; Bianchi and MacDonald, 2002; Chang et al., 2002). In general, desensitization of GABA-A plays an important role in regulating synaptic inhibition and neurotransmitter release. However, the role it plays during embryonic neurogenesis, a period when FMRP is critical, is less clear. Owens et al. found that early cortical neurons display less desensitization than more mature neurons, and that GABAA receptors are activated in a paracrine mechanism which does not involve synaptic activation (Owens et al., 1999). These results suggest that GABA plays extra-synaptic roles during neuronal maturation that are not yet fully understood More importantly, the role FMRP could play in the kinetics of GABA-A desensitization has not been established yet. Our study provides the first evidence for an FMRP-dependent maturation of GABA responses in human neurons developing in-vitro.

\section{Abnormal Expression of Specific GABA-A Subunits in FX Human Neurons}

The functional analysis of GABA response in WT and FX invitro human neurons led us to conclude that subunits in the GABA-A receptor could be abnormally expressed in FX neurons. We found that the $\alpha 1$ subunit was not expressed in WT nor in FX neurons. Furthermore, there was no significant difference between WT and FX neurons in the expression of $\gamma 2 s$ and $\delta$ subunits. However, a significant increase in $\alpha 2$ expression and a robust reduction in the expression of $\beta 2$ subunit were found in FX neurons as compared to WT, which could potentially explain the immature phenotype observed in FX neurons. A similar response to GABA, showing desensitization and lack of reactivity to bicuculline (similar to "type 2") was shown by Maddox et al. in normal human dorsal root ganglion cells (DRGs) from adult samples, but not in embryonic counterparts (Maddox et al., 2004). In that study, and ours, a correlation between neuronal immaturity and reduced expression of the GABA-A $\beta 2$ subunit was observed. It is important to note that both studies, Maddox et al. and the current report, analyzed mRNA levels of GABA-A subunits, rather than protein levels. Therefore, any conclusion on the molecular conformation of the actual receptor could be made only under the assumption that changes in transcription directly reflect changes in translation, which might not be necessarily so. Taken together, these data suggest that FMRP plays an important role in the regulation of the development of the GABAergic system in early human neurons.

In rodents, an increase in $\alpha 2$ expression has been linked with immature GABA responses characterized by slow decay in developing postnatal thalamic neurons (Okada et al., 2000). After maturation however, higher expression of $\alpha 1$, lower expression of $\alpha 2$ and faster decay are observed in the same neurons, indicating that $\alpha 2$ is probably responsible for slower decay. The same slow decay has been reported in-vitro in HEK cells following overexpression of $\alpha 2$, resulting in a $\sim 10$-fold slower decay for $\alpha 2$ over $\alpha 1$ (Dixon et al., 2014). In contrast, two different studies concluded that the slow decay component in GABA responses is a result of $\alpha 3$ expression (Geracitano et al., 2012; Labrakakis et al., 2014). Here we suggest that, in accordance to previous studies, the increase in $\alpha 2$ expression in FX neurons, which were predominantly "type-2," could be responsible for the slower decay in GABA responses in these neurons. A hypothesis can be formulated by which FMRP plays a role in the downregulation of $\alpha 2$ subunit during neuronal maturation. In FXS, where FMRP expression is lost, $\alpha 2$ remains abnormally high, impairing maturation. However, in order to test this hypothesis, more experiments need to be conducted.

\section{SUMMARY}

The findings of the present study have never been reported in the developing brain of the $f \mathrm{mrl}^{-/-}$mouse, and highlight the potential significance of the FX-hESCs system in modeling FXS. However, more studies are necessary to further explore the role of FMRP in the GABAergic developmental switch and its contribution to the pathology of FXS.

\section{AUTHOR CONTRIBUTIONS}

MT designed and performed the experiments, collected and analyzed the data, wrote the manuscript. MS designed the experiments, provided materials and funding, analyzed the data, wrote edited the manuscript. DB designed the experiments, provided materials and funding, analyzed the data, wrote and edited the manuscript.

\section{FUNDING}

This study was supported by the National Network of Excellency (NNE) in Neuroscience from TEVA Pharmaceuticals Ltd and by a grant from the Israel Ministry of Health.

\section{SUPPLEMENTARY MATERIAL}

The Supplementary Material for this article can be found online at: http://journal.frontiersin.org/article/10.3389/fncel. 2016.00121 


\section{REFERENCES}

Adusei, D. C., Pacey, L. K., Chen, D., and Hampson, D. R. (2010). Early developmental alterations in GABAergic protein expression in fragile X knockout mice. Neuropharmacology 59, 167-171. doi: 10.1016/j.neuropharm.2010.05.002

Bagni, C., and Oostra, B. A. (2013). Fragile X syndrome: from protein function to therapy. Am. J. Med. Genet. A 161, 2809-2821. doi: 10.1002/ajmg.a.36241

Bear, M. F., Huber, K. M., and Warren, S. T. (2004). The mGluR theory of fragile X mental retardation. Trends Neurosci. 27, 370-377. doi: 10.1016/j.tins.2004.04.009

Ben-Ari, Y. (2015). Is birth a critical period in the pathogenesis of autism spectrum disorders? Nat. Rev. Neurosci. 16, 498-505. doi: 10.1038/nrn3956

Ben-Ari, Y., Khalilov, I., Kahle, K. T., and Cherubini, E. (2012). The GABA excitatory/inhibitory shift in brain maturation and neurological disorders. Neuroscientist 18, 467-486. doi: 10.1177/1073858412438697

Bianchi, M. T., and MacDonald, R. L. (2002). Slow phases of GABA(A) receptor desensitization: structural determinants and possible relevance for synaptic function. J. Physiol. 544, 3-18. doi: 10.1113/jphysiol.2002.020255

Braat, S., D'Hulst, C., Heulens, I., De Rubeis, S., Mientjes, E., Nelson, D. L., et al. (2015). The $\mathrm{GABA}_{\mathrm{A}}$ receptor is an FMRP target with therapeutic potential in fragile X syndrome. Cell Cycle 14, 2985-2995. doi: $10.4161 / 15384101.2014 .989114$

Braat, S., and Kooy, R. F. (2015). Insights into GABA ergic system deficits in fragile X syndrome lead to clinical trials. Neuropharmacology 88, 48-54. doi: 10.1016/j.neuropharm.2014.06.028

Chang, Y., Ghansah, E., Chen, Y., Ye, J., and Weiss, D. S. (2002). Desensitization mechanism of GABA receptors revealed by single oocyte binding and receptor function. J. Neurosci 22, 7982-7990.

D'Hulst, C., and Kooy, R. F. (2009). Fragile X syndrome: from molecular genetics to therapy. J. Med. Genet. 46, 577-584. doi: 10.1136/jmg.2008.064667

Dixon, C., Sah, P., Lynch, J. W., and Keramidas, A. (2014). GABAA receptor alpha and gamma subunits shape synaptic currents via different mechanisms. J. Biol. Chem. 289, 5399-5411. doi: 10.1074/jbc.M113.514695

Eiges, R., Urbach, A., Malcov, M., Frumkin, T., Schwartz, T., Amit, A., et al. (2007). Developmental study of fragile X syndrome using human embryonic stem cells derived from preimplantation genetically diagnosed embryos. Cell Stem Cell 1, 568-577. doi: 10.1016/j.stem.2007.09.001

Geracitano, R., Fischer, D., Kasugai, Y., Ferraguti, F., and Capogna, M. (2012). Functional expression of the GABA(A) receptor alpha2 and alpha3 subunits at synapses between intercalated medial paracapsular neurons of mouse amygdala. Front. Neural Circuits 6:32. doi: 10.3389/fncir.2012. 00032

Guo, W., Allan, A. M., Zong, R., Zhang, L., Johnson, E. B., Schaller, E. G., et al. (2011). Ablation of Fmrp in adult neural stem cells disrupts hippocampusdependent learning. Nat. Med. 17, 559-565. doi: 10.1038/nm.2336

He, Q., Nomura, T., Xu, J., and Contractor, A. (2014). The developmental switch in GABA polarity is delayed in fragile X mice. J. Neurosci. 34, 446-450. doi: 10.1523/JNEUROSCI.4447-13.2014

Jones, M. V., and Westbrook, G. L. (1995). Desensitized states prolong GABAA channel responses to brief agonist pulses. Neuron 15, 181-191.

Kratovac, S., and Corbin, J. G. (2013). Developmental changes in expression of inhibitory neuronal proteins in the Fragile $\mathrm{X}$ Syndrome mouse basolateral amygdala. Brain Res. 1537, 69-78. doi: 10.1016/j.brainres.2013. 08.052

Labrakakis, C., Rudolph, U., and De Koninck, Y. (2014). The heterogeneity in $\mathrm{GABA}_{\mathrm{A}}$ receptor-mediated IPSC kinetics reflects heterogeneity of subunit composition among inhibitory and excitatory interneurons in spinal lamina II. Front. Cell. Neurosci. 8:424. doi: 10.3389/fncel.2014.00424
Luo, Y., Shan, G., Guo, W., Smrt, R. D., Johnson, E. B., Li, X., et al. (2010). Fragile $\mathrm{x}$ mental retardation protein regulates proliferation and differentiation of adult neural stem/progenitor cells. PLoS Genet. 6:e1000898. doi: 10.1371/journal.pgen.1000898

Maddox, F. N., Valeyev, A. Y., Poth, K., Holohean, A. M., Wood, P. M., Davidoff, R. A., et al. (2004). GABAA receptor subunit mRNA expression in cultured embryonic and adult human dorsal root ganglion neurons. Brain Res. Dev. Brain Res. 149, 143-151. doi: 10.1016/j.devbrainres.2004.01.001

Martinez-Torres, A., Demuro, A., and Miledi, R. (2000). GABArho 1/GABAAalpha 1 receptor chimeras to study receptor desensitization. Proc. Natl. Acad. Sci. U.S.A. 97, 3562-3566. doi: 10.1073/pnas.050582397

Okada, M., Onodera, K., Van Renterghem, C., Sieghart, W., and Takahashi, T. (2000). Functional correlation of GABA(A) receptor alpha subunits expression with the properties of IPSCs in the developing thalamus. J. Neurosci. 20, 2202-2208.

Owens, D. F., Liu, X., and Kriegstein, A. R. (1999). Changing properties of GABA(A) receptor-mediated signaling during early neocortical development. J. Neurophysiol. 82, 570-583.

Penagarikano, O., Mulle, J. G., and Warren, S. T. (2007). The pathophysiology of fragile x syndrome. Annu. Rev. Genomics Hum. Genet. 8, 109-129. doi: 10.1146/annurev.genom.8.080706.092249

Segal, M. (1993). GABA induces a unique rise of [Ca]i in cultured rat hippocampal neurons. Hippocampus 3, 229-238.

Sheridan, S. D., Theriault, K. M., Reis, S. A., Zhou, F., Madison, J. M., Daheron, L., et al. (2011). Epigenetic characterization of the FMR1 gene and aberrant neurodevelopment in human induced pluripotent stem cell models of fragile X syndrome. PLoS ONE 6:e26203. doi: 10.1371/journal.pone.0026203

Telias, M., Kuznitsov-Yanovsky, L., Segal, M., and Ben-Yosef, D. (2015a). Functional deficiencies in Fragile $\mathrm{X}$ neurons derived from human embryonic stem cells. J. Neurosci. 35, 15295-15306. doi: 10.1523/JNEUROSCI.031715.2015

Telias, M., Mayshar, Y., Amit, A., and Ben-Yosef, D. (2015b). Molecular mechanisms regulating impaired neurogenesis of fragile $\mathrm{X}$ syndrome human embryonic stem cells. Stem Cells Dev. 2, 2353-2365. doi: 10.1089/scd.2015.0220

Telias, M., Segal, M., and Ben-Yosef, D. (2013). Neural differentiation of Fragile X human Embryonic Stem Cells reveals abnormal patterns of development despite successful neurogenesis. Dev. Biol. 374, 32-45. doi: 10.1016/j.ydbio.2012.11.031

Telias, M., Segal, M., and Ben-Yosef, D. (2014). Electrical maturation of neurons derived from human embryonic stem cells. F1000Res. 3:196. doi: 10.12688/f1000research.4943.2

Willemsen, R., Bontekoe, C. J., Severijnen, L. A., and Oostra, B. A. (2002). Timing of the absence of FMR1 expression in full mutation chorionic villi. Hum. Genet. 110, 601-605. doi: 10.1007/s00439-002-0723-5

Conflict of Interest Statement: The authors declare that the research was conducted in the absence of any commercial or financial relationships that could be construed as a potential conflict of interest.

The reviewer LD and handling Editor declared a current collaboration and the handling Editor states that the process nevertheless met the standards of a fair and objective review.

Copyright (c) 2016 Telias, Segal and Ben-Yosef. This is an open-access article distributed under the terms of the Creative Commons Attribution License (CC BY). The use, distribution or reproduction in other forums is permitted, provided the original author(s) or licensor are credited and that the original publication in this journal is cited, in accordance with accepted academic practice. No use, distribution or reproduction is permitted which does not comply with these terms. 